NOTAS Y DISCUSIONES

\title{
Nota sobre la recepción del análisis filosófico en América Latina
}

\author{
FERNANDO SALMERÓN \\ Instituto de Investigaciones Filosóficas \\ Universidad Nacional Autónoma de México
}

\section{Primera parte}

1. El título de esta nota $y$, por supuesto, también su extension, obedecen con exactitud al pedido de los editores de Isegoría. La nota misma, sin embargo, no puede responder a todas las cuestiones que podrian plantearse a partix del título; tampoco pretende reunir toda la información pertinente sobre el amplio territorio que en el se señala. Las cuestiones históricas de fondo, como las propiamente filosóficas, no llegan a ser formuladas, en provecho de una propuesta para interpretar un panorama, que en ningún punto quiere ser exhaustivo, sino que destaca algunas líneas relevantes de la actividad filosófica en los países latinoamericanos en un cierto momento. Y desde luego, da por sentado que tal interpretación sólo tendría el respaldo necesario si contara con una visión integral de la historia de las ideas en América Latina durante el siglo que está por terminar -una historia todavía no escrita. ${ }^{1}$

Cabe todavía otra consideración previa: el trazo de estas líneas generales estará subrayado según se contempla aquel panorama - y esto, seguramente, contiene también marcas involuntarias que derivan de la propia experiencia. El autor desconoce mucho más, por ejemplo, de la vida filosófica del Brasil, que de la de otros países como Argentina o Perú, $y$, por otra parte, tiene mucho más cercana la de México, lo que ha permitido, en la segunda parte, introducir un tratamiento diverso para este caso. Semejantes desequilibrios en la información, que anularían un trabajo de otra indole, parecen menos graves cuando su función se reduce a ilustrar los rasgos de un paisaje -para lo que cuenta mejor un testimonio que el análisis de una obra importante.

Finalmente habría que decir que la expresión "filosofía analítica" -bajo cuyo rótulo no se reconocerían muchas de las personas nombradas-, se toma aquí en un sentido muy amplio, que alcanza desde una cierta manera de ver los problemas de la filosofía de la ciencia y los desarrollos de la lógica moderna, hasta la consideración filosófica del lenguaje y el análisis conceptual propiamente dicho. Algo así como de Viena a Cambrigde - $\mathrm{e}$ incluso al Oxford de los años sesenta.

2. El empirismo lógico, tal como surgió en el círculo de Viena, fue visto en- 
tre nosotros como el elemento renovador de una vieja polémica antipositivista. Un pequeño libro de Antonio Caso, publicado en México en 1941, reproduce un curso de historia del positivismo, desde Comte y Mill hasta el círculo de Viena, y dedica scndos capítulos intermedios a Mach y a Poincaré, hasta culminar con lo que Caso llama "el positivismo críticon, en oposición a la fenomenología de Husserl y de sus discípulos. Una oposición de dos corrientes filosóficas que, en otro lugar, el mismo autor señala como wel debate más importante y grandioso del pensamiento contemporáneo». En la reseña de ese pequeño libro, que José Gaos escribió unos meses después, recordó la polémica Husserl-Schlick y subrayó como mérito del maestro mexicano el haber utilizado la fenomenología anterior al neopositivismo en contra de las tesis de esta posición filosófica.

En verdad, el juicio de Caso acerca de las dimensiones del debate, sobre todo si se piensa en polémicas explícitas, podría no ser correcto en términos de filosofía europea. Pero en América Latina -toda proporción guardada en cuanto a dimensiones y aunque tampoco se hubieran dado demasiadas controversias teóricas-, habría que reconocer que la filosofía analítica fue recibida en los años posteriores al texto de Caso precisamente por los fenomenólogos -y no siempre sin conflicto-. De la propia cátedra de Caso, uno de los alumnos de las últimas generaciones, Nicolás Molina Flores -posteriormente traductor de Carnap y de Ayer- optó por el empirismo lógico y vino a ser, desde los años cuarenta, el primer defensor mexicano de esa corriente filosófica.

Lo dicho en relación con los fenomenólogos no indica que no se trabajara en esos años en filosofía y en historia de la ciencia -los libros de
García Bacca, algunos preparados en Ecuador pero publicados en México, que coinciden en fechas con el de Caso, prueban lo contrario. Pero prueban también que, al menos en estos dos países, es seguro que no fue por la ciencia, ni por la lógica, que surgió el interés en el análisis filosófico.

3. En los primeros meses de 1945, Gaos publicó un comentario en que saludaba la aparición de la primera revista hispanoamericana "dedicada exclusivamente a la filosofías. Se trataba de Minerva. Revista Continental de Filosofía, que el año anterior había empezado a editar Mario Bunge en Buenos Aires, junto con los Cuadernos de la misma revista, alguno de los cuales también comentó Gaos, como el escrito por Risieri Frondizi.

A pesar de que la revista tuvo una vida breve y de que dio cabida a autores de las más diversas corrientes filosóficas, su orientación general hacía patente una voluntad de alejamiento de la tradición dominante en las universidades latinoamericanas, en la década de los años cuarenta: la de Ortega y la filosofía de lengua alemana. $Y$ publicó además, en uno de sus primeros números, un artículo de Hans A. Lindemann sobre el círculo de Viena. Lindemann, entonces residente en Buenos Aires, habra seguido cstudios en Austria y tenía una visión de primera mano de las actividades del cínculo y de sus controversias internas. Esta anécdota de vida, obliga a recordar el ejemplo de Schajowicz: Ludwig Schajowicz, con una experiencia filosófica semejante en Viena, a su llegada a la Universidad de Puerto Rico, hubo de cambiar la orientación de sus preocupaciones ante la solidez de la tradición dominante $y$, ni su tarea docente, ni su obra escrita, reflejaron aquel interés inicial.

En Buenos Aires, sin embargo, las 
cosas marcharon de otra manera. Bunge, que vería del campo de la ciencia y trabajaba en física teórica, entró de lleno en los temas filosóficos y, en 1956, llegó a hacerse cargo de la cátedra de filosofía de la ciencia, desde donde desarrollo una amplia labor no limitada a su propio país durante casi una década -hasta que la situación política le llevó a continuar su carrera en el exterior. En 1959, la Universidad de Harvard publicó en inglés su libro Causalidad. El principio de causalidad en la ciencia moderna, la primera obra escrita en América Latina sobre estas materias, desde un punto de vista que podemos llamar analítico. Al año siguiente apareció en español la Antologia semántica, compilada por el propio Bunge, que incluía textos de Russel, Carnap, Hempel, Tarski, Quine, Goodman y Max Black, entre otros. Oportunidad para que el compilador distinguiera entre varias corrientes del análisis filosófico y precisara su adhesión en favor de aquella de origen neopositivista y neopragmático, al tiempo que señalaba también su desinterés por el análisis del lenguaje ordinario. Pero, en todo caso, defendía una posición abierta a todos los temas tradicionales de la lógica, la teoría del conocimiento y la ontología.

La formación de Frondizi era diferente, y también lo fue su influencia. Había estudiado en los Estados Unidos, primero en Harvard -con Whitehead, C.I, Lewis y R.B. Perry-, y luego en Michigan - con Sellars. Siempre se consideró a sí mismo como un empirista - defensor de un uempirismo humanistan-, y de hecho fue un severo crítico de las tendencias más ciencistas y técnicas de la filosofía analítica; pero su obra representa, en temas y estilo, un rechazo de la tradición dominante, influida por el pensamiento de lengua alemana. Frondizi enseñó en Venezuela y Puerto Rico y, finalmente -durante su último exilio político-, en los Estados Unidos, donde murió en 1983. Pero los años que trabajó en Argentina - aproximadamente una década, entre 1956 y 1966 - constituyen un período de recuperación de la vida académica de ese país, y coinciden con el ingreso a las universidades de los filósofos de orientación analítica.

4. No es exagerado decir que, en los años posteriores a la aparición de $M$ inerva, justo antes de la década que se acaba de señalar, los argentinos interesados en filosofía analítica tuvieron que trabajar en círculos y asociaciones ajenos a las universidades públicas. A manera de ejemplos de instituciones y personas, se pueden citar: el Grupo Argentino de Academia Intemacional de Historia y Filosofía de la Ciencia, al que pertenecía Julio Rey Pastor; el Instituto Libre de Estudios Superiores, en que trabajaba Gregorio Klimovsky; y el Circulo Filosófico de Buenos Aires, presidido entonces por Bunge. La única excepción parece haber sido la de Carlos Cossio, un filosofo del derecho de formación fenomenológica, $y$ por añadidura crítico de la filosofía analítica, que organizó en su seminario de la Universidad de Buenos Aires, estudios de lógica modal y de metodología de las ciencias deductivas.

Un discípulo de Cossio, Ambrosio Gioja, le sucedió en la cátedra en 1956. Tenia una obra escrita menos importante que la de su maestro y, sin embargo, ejerció una mayor influencia sobre los jóvenes interesados en filosofía del derecho. Gioja era también fenomenólogo, pero su dedicación a los clásicos -sobre todo a Kant-, y su interés por los contemporáneos -en especial Kelsen y Wittgenstein-, hicieron del Instituto de Filosofía del Derecho y de la revista Notas de Filosofía del Derecho, núcleos de gran actividad filosófica. La 
revista sobrevivió poco tiempo -entre 1964 y $1969-$ - y Gioja murió prematuramente, pero en esos años visitaron el Instituto algunos filósofos extranjeros: Alf Ross, Strawson, von Wright, Chaim Perelman y Ulrich Klug; además de los latinoamericanos Eduardo Garcia Maynez y Héctor-Neri Castañeda. El grupo de jóvenes que entonces se acerco al Instituto, ha venido a constituir la llamada "primera generación» de filósofos argentinos del derecho, de orientaciớn analítica: Carlos Alchourrón, Ėugerio Bulygin, Jorge A. Bacqué, Genaro Carrió, Ernesto Garzón Valdés, Eduardo Rabossi, Roberto Vernengo, María Isabel Azaretto y José Manuel Vilanova. Posteriormente sobrevino la «segunda generación", no menos nutrida que la anterior, entre quienes se cuentan CarIos Nino, Martín Farrel, Antonio Martino, Ricardo Caracciolo y Ricardo Guibourg. Todos ellos - los de ambas generaciones-, con una producción notable, reconocida más allá de las fronteras de su propio país. Lo que, por otra parte, no significa la completa unidad de temas y procedimientos: algunos han preferido las técnicas del análisis del lenguaje ordinario, como en el caso de Carrió, cuyo primer libro en esta dirección es Notas sobre derecho $y$ lenguaje, de 1965. Otros, las técnicas lógico-formales, como Alchourtón y Bulygin, autores de Normative Systems, publicado en 1971 y de una Introduccion a la metodologia de las ciencias jurídicas y sociales, de 1974. Sobre todo en la última década, varios de los nombrados han escrito obras de ética y numerosos ensayos sobre problemas morales y políticos de orden práctico.

Las limitaciones de espacio impuestas a esta nota, impiden dar cuenta de la producción filosófica de este grupo, pero no son excusa para algunas indicaciones generales y autorizan a no repetir información que alli puede encon- trarse. La primera consiste en remitir al lector al volumen antologico Argentinische Rechtstheorie und Rechtsphilosophie heute, publicado en Berlín, en 1987, por Garzón Valdés y Eugenio Bulygin, que además de ofrecer textos ejemplares, informa sobre las publicaciones de un buen número de los autores nombrados. Otra sugerencia consiste en acudir a sendas entrevistas a Genaro Carrió y a Garzón Valdés, publicadas en Doxa, los cuadernos de filosofía del derecho de la Universidad de Alicante, que dan un testimonio de primera mano sobre el ambiente filosófico de buenos Aires - y de la circunstancia política, tan favorable a los exilios permanentes o pasajeros. Pero no sólo de Buenos Aires, porque en un momento, la presencia de la filosofía analítica alcanzó a otras universidades como la de Tucumán y, principalmente, la de Córdoba, con la llegada de Garzón Valdés en 1958 -de vuelta de Alemania-, como profesor de filosofía del derecho; y de Andrés Raggio como profesor de lógica y de filosofía de la ciencia.

Las sugerencias anteriores, especialmente la lectura de las entrevistas, permitirán constatar también la fuerza decisiva de la vida filosófica local en la orientación de los protagonistas, en ocasiones por encima de la experiencia en las universidades europeas o en los Estados Unidos; además de aportar información sobre la labor de editores y traductores de algunos de los nombrados - Carrió, Rabossi y Garzón Valdés, por ejemplo-, en los años en que las editoriales argentinas, una de las cuales fue fundada por Frondizi, abrieron sus puertas a los autores de filosofía analítica. Los mismos años en los que la editorial Paidos, inició la Biblioteca de Filosofía dirigida por Gregorio Klimovsky y Saad Chedid. De Garzón Valdés habría que hacer una mención especial, porque a él se debe el conoci- 
miento en lengua española de buena parte de la nueva filosofía alemana de orientación analítica.

5. Klimovsky desempeño, en filosofía de la lógica y del lenguaje, un papel semejante al de Gioja en filosofía del derecho. Primero en organizaciones privadas y, desde 1956, en la cátedra de lógica de la Universidad de Buenos Aires - con las obligadas interrupciones por causa de los acontecimientos políticos. Pero en uno u otros sitios - tam bien en la Universidad de la Plata-, ha podido sostener su enseñanza por un largo período y formado varias generaciones de discípulos, a quienes ha impulsado además con su propia obra escrita sobre método deductivo y estructura y validez de las teorias científicas. Y a diferencia de Gioja, ha estado comprometido - desde 1943-, con la tradición empirista y el análisis filosófico. Todavía en 1964, Klomovsky escribió unas páginas de tono polémico, contra el contexto filosófico argentino impermeable al análisis. Esas páginas sirvieron de prólogo al líbro de uno de sus discípulos, miembro del grupo que podríamos llamar la "primera generación» argentina de filosofos del lenguaje de orientación analítica. El libro al que se acaba de aludir, Formas lógicas, realidad y significado, de Thomas Moro Simpson, era seguramente el primer libro escrito por un autor de lengua española sobre filosofía de la lógica, que discutía tesis de Russel, Frege, Church, Quine y Strawson. Años después, Simpson dio a la imprenta una cuidadosa antología de Semántica filosófica, que es modelo en su género.

Una segunda generación de jóvenes dedicados a la lógica, filosofía de la ciencia y del lenguaje, formados inicialmente en Buenos Aires, ha contribuido a mantener la actividad filosófica -aunque no todos han permaneci- do en el país. Alberto Coffa, que murió joven, había pasado a los Estados Unidos; allí reside también Ricardo Gómez; Raúl Orayen se incorporó definitivamente a la Universidad Nacional de México. Pero continúan en Argentina, entre otros, Juan Rodríguez Larreta, Alberto Moretti, Félix Gustavo Schuster y Cecilia Hidalgo. Este último grupo, junto con Klimovsky, Simpson y los nombrados por su dedicación al derecho y a la ética que residen en Argentina, fundaron en 1972, bajo la presidencia de Rabossi, la Sociedad Argentina de Análisis Filosófico, que no es solamente una agrupación de discusiones libres, sino un centro que imparte enseñanza de postgrado en filosofía. Desde 1981, la Sociedad publica semestralmente Análisis Filosófico, una revista que mantiene viva la tradición analítica; aunque esa tradición - y por supuesto sus defensores-, tienen un lugar habitual en las otras revistas argentinas especializadas en filosofia: la Revista Latinoamericana de Filosofia, que edita el Centro de Investigaciones Filosóficas; y Cuadernos de Ética, publicada por la Asociación Argentina de Investigaciones Éticas.

6. Las relaciones culturales de Argentina y Uruguay sicmpre han sido cercanas. Al menos desde 1956 existía la Agrupación Rioplatense de Lógica y Filosofía Científica, que reunía nacionales de los dos países. Sin embargo, en Uruguay hay que registrar un antecedente de excepción, el caso de Carlos Vaz Ferreira, cuyo trabajo no parece haber sido continuado por sus discipulos, la mayor parte de los cuales pasó a la fenomenología o a la historia de las ideas. Vaz Ferreira murió en 1958, pero a pesar de su interés por las filosofías de la vida no dejó de ser un empirista, influido por Stuart Mill, y cuya preocupación por los problemas concretos de 
la educación le llevó a una reforma de la lógica y a una manera personal de enfrentar el lenguaje ordinario, que obliga a considerarlo -en esta porción de su obra-como un antecedente directo del análisis filosófico.

Al fin de los años cincuenta, Ezra Heymann - que actualmente radica en Venezuela, adscrito a la Universidad Simón Bolivar-, enseñaba lógica en Montevideo y dio a conocer a Frege y a Austin, entre otros autores, gracias a su relación con Raggio, a quien había seguido en la Universidad de Córdoba. En ese tiempo, el predominio de la fenomenología, en especial de Heidegger, junto con el creciente desarrollo de los estudios latinoamericanos de historia de las ideas, apenas dejaban lugar a otras tareas.

Por esas fechas, Mario Otero, que habia pasado un tiempo en la Universidad de Nueva York, en Buffalo, con Marvin Farber, viajo a Buenos Aires a trabajar su tesis de doctorado con Bunge; de allí pasó a París y nuevamente a los Estados Unidos, donde siguió cursos de Quine y Putram. Parte de su obra escrita sobre historia de la lógica y filosofía de la ciencia, la realizó en México, en el Instituto de Investigaciones Filosóficas, en años de exilio, pero con el restablecimiento de la democracia en Uruguay, Otero volvió a Montevideo, adscrito a la Universidad de la República. El país, sin embargo, perdió a Hcymann y perdió también a la generación siguiente: Javier Sasso, Eduardo Piacenza y Carlos Pereda; los dos primeros permanecieron en Venezuela; el útimo en México. Los tres se prepararon en universidades europeas y trabajan sobre filosofía contemporánea, historia de la lógica, filosofía del lenguaje y teoría de la argumentación; pero en forma muy abierta a todas las direcciones del pensamiento contemporáneo, que muestra a la vez el dominio de las técnicas del análisisis.
7. El caso de Chile parece diferente $y$ no puede entenderse sin admitir un cierto aislamiento. La enseñanza de la filosofía en este país, desde mediados del siglo y una vez desaparecida la influencia del positivismo decimonónico, parece orientada sobre todo al dominio de los grandes clásicos de la historia de la filosofía. Y, en un cierto momento, se puede tener la impresión de que el campo quedó repartido entre tomistas y heideggerianos. Sin embargo, los estudios de lógica llegaron a muy aito nivel, en las publicaciones y en la ensenanza, con Juan Rivano, Gerold Stahl y Rolando Chuaqui - hasta que los interrumpió el golpe militar de 1973. Fundamentalmente en atención a estos tra. bajos -no exclusivamente a ellos, por supuesto-, cabria conjeturar que la recepción de la filosofía anatítica en Chile se debe sobre todo a los lógicos.

Lo anterior se debe matizar teniendo en cuenta, entre otras, precisiones como las que siguen. Las publicaciones de Roberto Torreti hasta poco antes de su salida de Chile y su incorporación a la Universidad de Puerto Rico, al comienzo de los años setenta, son principalmente de historia de la filosofía -dominadas por su excelente libro sobre Kant. Solamente después han visto la luz sus estudios de historia y filosofía de la ciencia y su trabajo sobre Wittgenstein. Pero en Puerto Rico ha contribuido a reorientar los estudios filosóficos, como puede constatar el lector de la revista Diálogos. En cierto modo, ha resultado inversa la trayectoria de otro chileno de la generación mấs joven: Alfonso GómezLobo, a su regreso de Heildelberg en que estudió con Ernst Tugendhat, publicó una antología de Frege con Siete escritos sobre lógica y semántica, lo que significaba en Chile la apertura de un nuevo territorio de investigación. Después preparó un volumen con textos 
del mismo Frege, Russell, Strawson y otros autores de orientación analítica, que ya no pudo ser publicado por causa del golpe militar. Su traslado a Washington y su incorporación a la Universidad de Georgetown, le han llevado a publicaciones de historia de la filosofía griega -aunque en algunas de ellas sea muy notable su preocupación por los problemas de la ética analítica.

8. En el Perú, la presencia del análisis se puede determinar con bastante exactitud a partir de la obra de dos de las mayores figuras filosóficas de ese país en lo que va de siglo: Francisco Miró Quesada y Augusto Salazar Bondy. La obra de ambos es nutrida y compleja - más vasta la del primero, el mayor en edad; menos la del segundo, fallecido además a edad relativamente temprana, en 1974. Pero hay un cierto paralelismo en el desarrollo de los dos autores que es el que vale la pena destacar.

Por diversas vias, ambos lograron una formación en filosofía europea continental y se iniciaron propiamente como fenomenólogos. Miró Quesada, después de un curso de moral, publicó El sentido del movimiento fenomenológi$c o$, pero enseguida derivó a cuestiones de lógica y filosofía de las matemáticas - dentro de una línea de investigación que puede considerarse analítica y que prácticamente no ha abandonado hasta ahora, desde la década de los años cuarenta. Al margen de esa permanente dedicación, centrada en los lenguajes formales, la obra de este autor se extiende al campo del pensamiento políti$\mathrm{co}$, en que igualmente viene a ser pionero en los países latinoamericanos. Un ejemplo son sus publicaciones del comienzo de los años sesenta, en que se comprometió con un movimiento político, en defensa de una actitud humanista fundada en postulados morales que, ajena al maxxismo, proponia algunas medidas socialistas ajustadas a las necesidades de su país. Son escritos por completo confiados en el conocimiento de las ciencias empíricas para los asuntos sociales; $y$, a la vez, en la eficacia del análisis para la clarificación de valores postulados, sin otro apoyo que una decisión a partir de sentimientos de simpatía y solidaridad. Una orientación general que se puede documentar en la tradición del empirismo y del racionalismo no dogmático. En cambio, ya no parecen caer dentro de la misma línea filosófica los escritos más recientes de Miró Quesada: los publicados a partir de la década de los affos setenta sobre filosofia de la historia y sobre historia de las ideas latinoamericanas.

Los primeros trabajos de Salazar Bondy -en los años ciencuenta-, son dos estudios sobre la filosofía en el Perú y su tesis de doctorado, una investigación fenomenológica sobre la distinción entre ser ideal y ser irreal, que se publicó posteriormente. Habia estudiado en México con José Gaos, entre 1948 y 1950, y tal vez de alli derivó esa doble inclinación intelectual, que no habria de abandonar nunca totalmente, a la historia de las ideas y a la terminología como método. Tras un tiempo de estancia en Europa, Salazar fortalece su formación en varias direcciones y comienza una etapa muy productiva: comprometido con un nuevo movimiento político peruano, que se inicia al final de los años sesenta, escribe sobre filosofía de la cultura y trabaja en un gran proyecto de educación que propone para su país un socialismo humanista - más cercano a Sartre, por ejemplo, que a un marxismo ortodoxo. Pero a la vez avanza filosóficamente: desde 1961, había empezado a publicar sobre filosofia moral británica y había traducido a Moore y a Wittgenstein, 
hasta reunit una serie de ensayos de analisis del lenguaje valorativo, que aparecieron como libro en 1971, en Santiago de Chile.

Desde el angulo que nos interesa, la aportación de Salazar es muy precisa en dos puntos. En primer lugar, entiende el análisis conceptual como inseparable de la consideración de la experiencia $y$, en este sentido, como un complemento del análisis fenomenológico. Esto le permite alcanzar un punto de vista previo a toda interpretación -que llama trascendental-, y que es a la vez condición de posibilidad del mundo objetivo de la praxis y de su comprensión y construcción racionales. En segundo lugar, defiende la función instrumental y crítica de la filosofía y la necesidad de su mejor equipamiento metodológico y conceptual, sin el cual no es posible una conciencia veraz de la propia realidad, aunque advierte el peligro de un divorcio respecto de los problemas más urgentes y reclama un esfuerzo convergente de la filosofía con la investigación científica en el plano teórico, y con la acción política en el práctico. Sin embargo, en su libro póstumo sobre la reforma educativa peruana, reconoce de hecho como scparables a las ideologías, es decir, a las concepciones del mundo. $Y$ afirma con toda claridad su función orientadora de la acción individual, y su lugar central en la educación y en los movimientos sociales.

Lo mismo Salazar que Miró Quesada mantuvieron siempre una relación directa con Argentina y México. Desde 1967, ambos formaron parte del consejo editorial de la revista Critica. De la segunda generación que les sigue, más interesada al parecer en los nuevos desarrollos de la filosofia alemana, hay que distinguir - por sus publicaciones sobre filosofía de la ciencia-, a Alberto Cordero, que estudió en Oxford con
Rom Harré y actualmente enseña en Nueva York.

9. La presencia del análisis filosófico en el Brasil, obedece a condiciones propias que, sin embargo, cuando se contemplan en perspectiva histórica no difieren demasiado de las de los demás países latinoamericanos. En Brasil fue más fuerte la presencia del positivismo comteano al finalizar el siglo pasado; y más fuerte, también, la reacción en la primera mitad del $\mathrm{xx}$ : una reacción orientada por las filosofias europeas del continente, pero sobre todo por el tomismo $\rightarrow$ y por el nacionalismo que marcó el estudio de las ideas propias. Hacia fines de los años cuarenta, sin embargo, la Universidad de Sao Paulo inició, para su recién fundada Facultad de Filosofia, una práctica que resultó decisiva para cambiar el ambiente filosófico: la de invitar por largos periodos a p̀rofesores extranjeros; además de Martial Gueroult, que contribuyó a desarrollar la historia de la filosofia: Williard V.O. Quine que, en 1944, publicó en portugués El sentido de la nueva lógica; y poco después, Gilles Gastón Granger - convertido desde entonces en un visitante regular de las universidades brasileñas-, quien escribió directamente en portugués, en 1955, su Lógica y filosofía de las ciencias.

Como en otros países hispanoamericanos, los estudios de lógica, la historia de la filosofía dirigida hacia los clásicos del empirismo, y los enfoques propiamente analíticos de los problemas, no surgían como continuación del viejo positivismo, sino como un intento de innovación filosófica, que habría de prosperar en un ambiente intelectual adverso. Los estudios de lógica han alcanzado en el Brasil un amplio desarrollo, primero en las universidades de Sao Paulo y Campinas, y después en Porto Alegre, Brasilia y Rio de Janeiro. 
Su figura de mayor relieve internacional es Newton da Costa, un especialista en logicas paraconsistentes. En los estudios de historia de la filosofía habria que destacar al menos un par de ejemplos: Joao Paulo Monteiro, el editor de la revista Ciencia e Filosofia, que es a un tiempo una autoridad sobre Hume $y$ sobre cuestiones epistemológicas actuales. En 1975 publicó su libro Teoria, retórica, ideología. Oswaldo Porchat es el otro ejemplo: un especialista en Aristóteles que a su regreso de Berkeley - de estudiar en un programa de Tarsky-, se incorporó al nuevo Centro de Lógica, Epistemología e Historia de las Ciencias de la Universidad de Campinas. Un Centro de gran actividad, que desde 1977 edita la revista Manuscrito, y que patrocinó el primer Congreso Latinoamericano de Lógica. A ese centro se encuentran adscritos, entre otros filósofos de orientación analítica, Balthazar Barbosa Filho, Henrique López Dos Santos, Seljko Loparic y Marcelo Dascal. Este último, editor de varios libros de amplia circulación y autor de Leibniz. Language, Signs and Thought.

De esta manera, la filosofía analítica ha ganado su lugar dentro de la vida filosófica del país, a pesar de la oposición inicial; y a pesar de dificultades especiales como el golpe de cstado de 1964 y sus consecuencias para las universidades en los años inmediatamente posteriores. Actualmente, la lógica y el análisis no se enseñan sólo en Sao Paulo y Campinas, sino en casi todas las universidades brasileñas - sin excluir las católicas. La Pontificia Universidad Católica de Rio de Janeiro, por ejemplo, tiene un programa de doctorado en filosofía de la ciencia, dirigido por Oswaldo Chateaubriand, un discípulo de Quine; y sin excluir tampoco los institutos tecnológicos: Leónidas Hegenberg es el caso de un conocido lógico adscrito a uno de ellos.
10. Las influencias filosóficas dominantes en Venezuela, desde los años cuarenta y hasta el comienzo de los setenta, fueron las mismas que en México: primero Husserl y la fenomenología; después Heidegger y Sartre; y al fin de ese período, el marxismo - con un acento derivado de la lectura de Hegel. El maestro que vivió ese período, y de alguna manera dominó todos los cambios, fue García Bacca. Gaos estuvo en Caracas en alguna ocasión, pero no alteró ese orden. Frondizi, en cambio, que hizo a sus alumnos estudiar el empirismo inglés, dejo huella en algunos de ellos. La formación de Juan Nuño tuvo lugar en aquellos años y su obra publicada posteriormente repasa, casi de manera fotográfica, esas influencias dominantes: libros sobre Platón y Heidegger, sobre Sartre y el marxismo. Todos ellos severamente críticos a propósito de los autores contemporáneos. Pero Nuño además, al comienzo de la década de los años cincuenta, fue a Cambridge tras la huella de los empiristas y estuvo con Bochensky en Friburgo; a su regreso a Caracas se hizo cargo del seminario de filosofía moderna y de la cátedra de lógica. En su maestro García Bacca encontró aliento para su nueva empresa, y en los marxistas y metafísicos venezolanos, la resistencia que necesitaba su temperamento polémico.

En 1973, Nuño publicó un libro de lógica formal; pero desde 1965 había dado a la imprenta otro titulado Sentido de la filosofia contemporánea, que es el primer panorama sobre esta materia, escrito en un país latinoamericano, que confiere un lugar preferente al empirismo lógico y a la filosofía analítica. Un pequeño libro que describe, al lado de la persistencia de la metafísica, la vigencia de las ideologías - y exhibe también la pugna entre las corrientes del empirismo más apegadas a la ciencia y la filosofía del materialismo dia- 
léctico. Los ensayos de Nuño que discuten temas y autores contemporáneos, escritos en los años sesenta, aparecieron como libro en 1970; los posteriores a esa fecha fueron reunidos en el volumen Compromisos y desviaciones, en 1982. La lectura de los dos libros, que descubre una amplia perspectiva temática, deja ver además una línea de evolución del autor unida a los cambios en el ambiente filosofico de su país: es el transito gradual a un clima que permite el libre curso del análisis filosofico como moneda académica corriente -pero revela también el paso de los descubrimientos y del entusiasmo combativo, a un cierto desencanto.

Además de Nuño, trabajaron en Venezuela en la misma dirección, en esos años, otros autores no venezolanos, entre ellos el argentino Ernesto Battistella, quien publicó estudios sobre logica deóntica. El interés por la lógica juridica se extendió a los profesores de derecho, para quienes García Bacca tradujo, en 1961, el libro de Klug. Posteriormente, han trabajado también los venezolanos de la generación más joven: Rafael Burgos y Pedro Lluveres, entre los más conocidos por sus escri. tos. El último de cstos dos autores publicó, en 1976, Ciencia y escepticismo.

11. El proceso de recepción del análisis filosófico en Colombia, del estudio de las figuras contemporáneas del empirismo y de sus temas, parece iniciarse un poco más tarde que en otros países, pero todavía dentro de la década de los años sesenta. También su discusión resultó menos áspera, por ejemplo, que en Venezuela, aunque las posiciones desde las que se combatió fueron iguales -en primer lìgar, heideggerianas, en segundo lugar, marxistas. Pero en Colombia son las mismas revistas generales y sobre todo las mismas revistas especializadas - Ideas y Valores, Cua- demos de Filosofía y Letras-, las que han dado lugar a la presencia del análisis, al lado de las direcciones continentales de la filosofía europea, que se mantienen con harto vigor.

La tarea principal en Colombia, ha corrido a cargo de Rubén Sierra Mejía, inicialmente formado en el estudio de fenomenólogos como Max Scheler, Heidegger y Sartre. Pero a partir de 1966, en que se hizo cargo de la cátedra de Lógica en Bogotá, en la Universidad Nacional, emprendió el estudio de Russel, Frege y Carnap; y poco despućs inició cursos y seminarios sobre Popper, Austin y Strawson. Además de algunas traducciones -de Russel, Gödel y Koyré--, Sierra Mejía ha contribuido regularmente, en las revistas colombianas, con ensayos que después ha reunido en libros: el primero, de 1978; el último, de 1985, que lleva por título Apreciación de la filosofía analitica -y que es una muestra de la apertura de sus intereses intelectuales. Varios profesores más jóvenes, formados en Europa, han contribuido a que estas materias se extendieran a otras disciplinas - por ejemplo, psicología y linguística-, y además a casi todas las universidades colombianas. Publicaciones más recientes de Colombia, contadas sobre todo a partir de la visita de Ernst Tugendhat en 1984, parecen haber suavizado todavía más el diálogo entre los autores de la tradición heideggeriana y hermenéutica, y los interesados en los desamollos del análisis filosófico.

En otros paises latinoamericanos, la presencia del análisis filosófico ha sido más débil, pero no ausente por completo. De los centroamericanos, por ejemplo, es indispensable registrar el nombre de Claudio Gutiérrez, graduado en la Universidad de Chicago a mediados de los sesenta, profesor de lógica en la Universidad de Costa Rica y colabora- 
dor activo de revistas especializadas de los Estados Unidos, Argentina y México. Fuera de Costa Rica, sin embargo, la situación política ha sido extraordinariamente difícil los últimos años. En la Universidad de San Carlos, en Guatemala, Héctor Neri-Castañeda enseñó filosofía después de 1954, de vuelta de sus estudios en Estados Unidos e Inglaterra, hasta que se trasladó a la Universidad de Wayne y más tarde a Indiana, donde ha realizado toda su obra filosófica. Un antiguo colega de Castañeda, Rigoberto Juárez Paz, permaneció en la ciudad de Guatemala, adscrito a la Universidad Francisco Marroquín, una institución privada.

Castañeda hizo en Guatemala diversas publicaciones, desde su ensayo sobre la naturaleza de los problemas filosóficos y sus estudios de filosofía de la aritmética, hasta su pequeño libro $L a$ dialéctica de la conciencia de si mismo, editado por la Universidad de San Carlos en 1960, cuando ya era profesor de la Universidad del Estado y de Wayne. Todavía en 1957 publicó en Dianoia "Un sistema general de lógica normativa», firmado como profesor de San Carlos. El pequeño libro sobre la autoconciencia se presenta como una propuesta coherente $y$ no incompatible con los hechos, de una manera de ver las relaciones entre conjuntos de conceptos del lenguaje ordinario de lo mental. Contiene muchos de los temas y del estilo de argumentar y establecer distinciones de la obra posterior de Castañeda $y$, por supuesto, toda la bibliografía de lengua inglesa pertinente; por eso mismo llama la atención que el planteamiento inicial del problema se hace a partir de obras y autores corrientes en los países americanos de lengua española: Scheler, Heidegger y Sartre.

\section{Segunda parte}

12. De los antecedentes del análisis en México, quedaron dichas dos palabras en las primeras líneas de esta nota. Ahora conviene retomar la cuestión recordando otro texto de Gaos, leído como conferencia en el Colegio de México, el 15 de octubre de 1965, aunque no impreso sino dos años después en una colección de ensayos reunida por el propio autor, que fue su ultimo libro publicado en vida. Para 1965 babía cambiado en México - y en América Latina-, el panorama filosófico y, por primera vez de una manera directa, el maestro hace público lo que considera el contendio esencial de las discusiones privadas con sus propios discípulos. Lo hace ciertamente en un estilo enérgico y no exento de ironía, que empieza por adelantar, a propósito de otro contexto. la observación de que "la rebelión de los discípulos" ha sido siempre un motor de la historia de la filosofía. Pero lo que importa no es sólo su poco aprecio por lo que llama filosofías del empirismo lógico, positivismo lógico, filosofía científica y analítica, sino el reconocimiento de su auge notorio, su explicación del hecho y el punto al que dirige su argumento principal.

El auge de estas filosofías, le parece a Gaos que se debe en su parte decisiva, por otro lado inexplicable, al vacio que se ha producido en la filosofia alemana y en sus dependencias francesa e italiana. Después de Heidegger, Jaspers y Marcel, ya estériles — solamente Sartre le parece aún fecundo-, tiene la impresión de que a ninguno de ellos les sigue un continuador de estatura pareja. $\mathrm{Y}$ el hueco ha sido cubierto por las filosofías que encuentran respaldo en el ciencismo del pensamiento moderno. Esta misma preocupación por la ciencia, según Gaos, se conecta en estas filosofías en auge con la convicción de 
que será suficiente con que la filosofía deje de ser sistemática y pase a ser un mero análisis conceptual, para que se convierta en científica. Y su error consiste no tanto en esa convicción, como en suponer que la filosofía puede dejar de ser sistemática. Porque el mero análisis, insiste Gaos, descubre de inmediato que los conceptos que verdaderamente importan a la filosofía son de suyo sistemáticos, en el sentido de que entrañan necesariamente el sistema y conducen a él. Pretender una filosofía no sistemática es, por tanto, un contrasentido - no menos grave que pretender una filosofía científica $o$, simplemente, objetiva. Porque el sistema como tal $-\mathrm{y}$ justo por ser sistema-, ha de ser subjetivo, en el sentido de que no puede ser, con autenticidad, aceptado como verdadero por ningún sujeto distinto de su autor.

Sin ser paradójica, esta conclusión de Gaos en defensa de un rasgo de la filosofía clásica y tradicional, que idenlifica filosofía, sistema y concepción del mundo, no dejaba de ser decepcionante y de representar para sus discípulos una vía cerrada. Aunque en el interior de los sistemas personales Gaos había reconocido siempre una buena porción de elementos aprovechables por todos - por tanto, separables del conjunto y con valor intersubjetivo-m, justamente las partes fenomenologicas: las descripciones y los análisis acerca de los fenómenos de este mundo. Aquí no habría espacio para comentar el alcance de la obra escrita y docente de Gaos que, por otra parte, el autor de esta nota ha intentado en otro lugar. Pero es indispensable al menos registrar un par de noticias relacionadas con su interés por lógica y con su idea del método filosófico.

Aún en el tiempo en que García Máynez comenzó a ocuparse de problemas de lógica y de su aplicación a la estruc- tura de la norma jurídica, al arrancar la década de los años cincuenta, la enseñanza de aquella disciplina - al menos en los niveles superiores-, siguió siempre al libro de Pfänder, cuando no se trataba de escolástica o de la llamada lógica dialéctica. El primer libro editado en México que contribuyó a cambiar esa orientación, fue el de Ferrater Mora y Leblanc, cuyo manuscrito conoció Gaos según cuentan los propios autores. Gaos habia trabajado por ese cambio $\mathrm{y}$, con la colaboración de una discípula suya, trató de introducir en la Facultad de Filosofía de la Universidad Nacional, los manuales americanos empleados en el Mexico City College, donde prestaba sus servicios. El propio Gaos dictó un curso monográfico, con ayuda del libro de Lukasiewicz sobre la silogística de Aristóteles, que fuc probablemente uno de los primeros cursos de logica moderna que se impartieron en esa facultad.

En cuanto al método filosófico, Gaos era un fenomenológico consecuente y defendía como "la primera gran regla» de ese método, la de partir de las expresiones verbales que designan el fenómeno que se trata de estudiar, para elegir después el caso ejemplar y proceder al análisis descriptivo, conceptual y torminológico, hasta despejar lo esencial del fenómeno. Habia derivado del primer Heidegger la idea de que, en el desarrollo de la filosofía moderna, este procedimicnto husserliano se había integrado con el método trascendental de Kant y, posteriormente, con el hermenéutico de Dilthey. Pero también reconoció de una mancra expresa, en la discusión con sus discípulos, la posibilidad y conveniencia de incorporar el análisis conceptual de la filosofía analítica y los resultados de la nueva filosofía del lenguaje y de la lógica - a los que consideraba en gran parte aun paralelo anglosajón de la fenomenología 
gemánica». Reconocimiento que, interpretaciones aparte, pudo ser visto por los discípulos, en su momento, como un acercamiento del maestro hacia ellos.

Gaos había abandonado, al menos desde 1953, su apego al historicismo extremo y a la filosofía del primer $\mathbf{H e i -}$ degger. En años posteriores había publicado además reseñas en que afirmaba la imposibilidad de seguir al Heidegger tardío, y su relativo alejamiento de Ortega. Con lo cual era consecuente su explicación, a mediados de los años sesenta, acerca del auge de la filosofía analítica; pero era consecuente también la reacción de algunos de sus discípulos más cercanos. Antes de hablar de estos es indispensable hacer alguna indicación sobre otros antecedentes, para dar una idea del clima filosófico de esos años.

13. Podrían contarse como antecedentes indirectos, aunque ciertamente de peso muy escaso, algunas traducciones de libros de filosofía de la ciencia, como las que inició García Bacca a su paso por México; o las que más tarde promovió De Gortari. Algo semejante podría decirse de los cursos de Arturo Rosenblueth que, no obstante su importancia, tuvieron solamente difusión entre los círculos científicos, hasta la aparición, ya muy tardia, en 1970 y 1971, de sus dos libros. Menos repercusión aún tuvieron las publicaciones de la última epoca de la vida de Recasens Siches sobre argumentación y retórica, que discutian, por ejemplo a Perelman y a Toulmin, no tanto por estar destinadas a un público de juristas, sino ante todo por presentarse como una ofensiva contra la lógica formal.

García Máynez, en cambio, tomó el partido de la lógica formal, si bien tampoco tenía ninguna inclinación por las direcciones empiristas de la filosofía, ni por el análisis -y así lo reiteró en 1969. Su formación fue fundamentalmente fenomenológica - habia estudiado en México con Antonio Caso y en Berlín con Nicolai Hartmann-, y nunca abandonó esa orientación inicial, pero durante poco más de 20 años, contados a partir de 1939 , trabajó sobre ontología formal del derecho y lógica. Aunque su punto de partida era la axiomática valorativa de Scheler y Brentano, García Máynez no trataba de establecer principios de orden axiológico sino leyes lógicas sobre la validez o invalidez de las normas del derecho. Su primer libro de lógica jurídica sigue a Husserl y a Pfänder, pero sus libros posteriores sobre esta materia -el último es de 1964 , acusan un dominio de la logica moderna que va mucho más allá de sus primeros estudios, y el prestigio de los resultados fue decisivo para las generaciones más jóvenes. La obra de García Máynez fue comentada por Gaos y Castañeda en México; en el extranjero, por Bobbio y Kalinowrsky. Y él mismo había discutido la lógica modal de Von Wright en 1953.

Posteriormente, García Máynez volvió a los temas sistemáticos de la filosofía del derecho, a la historia de la filosofía griega y a las convicciones metafísicas de su juventud, pero permanecló su ejemplo de disciplina en el cumplimiento de un programa filosófico que contribuyó a descubrir un nuevo campo de investigación. Dentro de la Universidad Nacional, fundó, además, un centro de estudios que después pasó a ser el Instituto de Investigaciones Iilosóficas y, desde 1955, inició la publicación de Dianoia. Sobre este autor - y sobre otros que serán nombrados más adelante-, el lector hallará unas noticias, que no se van a repetir aquí, en la Introducción al volumen Philosophie und Rechtstherorie in México, editado por L. Olivé y F. Salmerón en 1989. 
Pero para integrar el cuadro o, más bien, las líneas que se quieren subrayar del cuadro de la filosofía en México, hacia la segunda mitad de la década de los años cincuenta, hace falta recordar a un investigador del Iristituto de Investigaciones Filosóficas, que fue también su consejero editorial en esos años: Robert S. Hartman. De origen alemán, Hartman había iniciado su formación en su propio país, donde tuvo ocasión de escuchar a Husserl y a Max Scheler. Después se trasladó a los Estados Unidos donde continuó sus estudios de lógica y de filosofia de la ciencia, de manera que pudo plantearse un programa que tenía cierto paralelismo con el inicial de García Máynez - pero en el campo de la axiología. En realidad se propuso elaborar un sistema lógico formal de los enunciados axiológicos, que no llegó a desarrollar íntegramente, pero que presentaba como un intento de axiomatizar fórmulas de G.E. Moore. No era, de ninguna manera, un filósofo analítico -en 1964 escribió un largo ensayo contra las teorías éticas de lo que, en ese momento, llamó la escuela de Oxford-, pero era un hombre enterado, y parte de su trabajo es un diálogo polémico con la filosofía contemporánea de lengua inglesa.

14. Hasta aquí los antecedentes. Porque en verdad, si dejamos a un lado el caso aislado, nombrado antes, de Molina Flores, no se puede hablar de filosofia analítica en México hasta 1959: el año en que, con motivo del centenario de Husserl, el Seminario de Filosofía Moderna de la Facultad de Filosofía de la Universidad Nacional celebró una sesión pública sobre el texto husserliano de 1910, La filosofía como ciencia rigu* rosa. El mismo año en que apareció la traducción castellana de Principia Ethica de Moore, realizada por Adolfo Gar- cra Diaz. Dos acontecimientos que se podrian enlazar si no hubiera limitaciones de espacio. Habrá que conformarse con decir que el traductor de Moore y uno de los miembros del seminario de filosofia moderna - Alejandro Rossi-. trabajaron cercanamente durante 1958 y 1959; y que ambos habían recibido una formación filosófica semejante - por cierto en condiciones diversaspero casi igual en cuanto a las materias de estudio: Hegel, Husserl y Heidegger.

El seminario de filosofía moderna era dirigido por José Gaos y estaba destinado exclusivamente a profesores de la Facultad. La propia Facultad publicó dos años después las ponencias de aquella sesión pública con unas notas sobre Husserl, escritas después de la reunión, por el director del seminario. Sobre estas notas y sobre el texto de Rossi, habrá que decir dos palabras.

El texto de Rossi caracteriza el ensayo de Husserl como un manifiesto filosófico: la confrontación con fílosofías que se conciben a sí mismas como concepciones del mundo; y la defensa del ideal de una disciplina como ciencia estricta; más una exposición de motivos de esta actitud filosófica, que la descripción ponderada de una situación histórica real. Mientras la concepción del mundo obedece a exigencias particulares inmediatas de explicación total y de salvación personal, la filosofía cientifica requiere una base teórica diferente y una manera de concebir su propia tarea como empresa de largo plazo. Una empresa, además, ligada a la posibilidad de un reino ideal de conceptos; susceptibles de ser analizados con todo rigor según cánones cientificos. De esta manera defiende. Rossi la contemporaneidad de Husserl y acaba por convertir sus tesis en un manifiesto filosófico propio, que anuncia una problemática no subordinada a exigencias espirituales inmediatas, un anuncio en 
que no faltan los nombres de Moore, Reichenbach, Wittgenstein y Feigl.

Las notas de Gaos no son una respuesta directa a Rossi, y apenas aluden a Villoro, otro de los miembros del seminario. Pero son una respuesta indirecta en la medida en que hacen una lectura pertinente del texto de Husserl, introducen sutiles distinciones para objetar el punto y, finalmente, establecen rangos de cientificidad para cada una de las disciplinas filosóficas. En cuanto a la que Gaos considera el núcleo último de la filosofía - la metafísica clásica-, rechaza la idea de su posible cientificidad y literalmente presenta esta idea como un monstruo de contradicciones. No obstante, concluye con la aceptación del método de la actual filosofía científica: porque parece proceder del análisis de los teoremas científicos $\mathrm{y}$ de los filosofemas mismos. Esto es sin disputa el buen método. Pero advierte enseguida que con ese método se obtiene una filosofia que no es la clásica filosofía metafísica, sino precisamente su condenación.

Me he detenido en estos dos textos, no tanto para ilustrar lo que podría llamarse la línea mexicana de continuidad - recepción y conflicto-, entre fenomenología y análisis, cuanto para dar fuerza a la conjetura enunciada al iniciar estas páginas que, en términos generales, quiere ser válida también para la mayor parte de los países de América Latina.

Por añadir más pruebas, podría detenerme en las circunstancias que concurrieron en la fundación de Critica, Revista Hispanoamericana de Filosofía y en el ajuste de sus propósitos, pero he relatado el caso en el informe de doce años del Instituto de Investigaciones Filosóficas, publicado en 1978, que me dispensa también de repetir otras noticias. Igualmente resultaría útil repasar los índices de Dianoia para se- guir el paso paulatino de la fenomenología al análisis. Porque la presentación del primer número de Crítica, en enero de 1967, aunque declare preferir "explicaciones que apelen a la descripción y al análisis», ya no mantiene huella de ese paso. Por el contrario, trata de acentuar los rasgos propios de una filosofía que se concibe a sí misma como análisis conceptual y como crítica, que quiere lograr una mayor precisión y claridad en sus argumentos; acercarse a la marcha actual de las ciencias y preocupaciones por su metodología. Todo esto, además, sin pretender representar ninguna escuela.

15. La obra escrita, a partir del comicnzo de la década de los años sesenta, por cada uno de los tres miembros del primer comité de dirección de Criti$c a$, viene a confirmar plenamente aquella continuidad. El primero de los cinco ensayos que Rossi reunió en Lenguaje y significado, su libro de 1969, se ocupa de las investigaciones lógicas de Husserl. Los ensayos posteriores tratan: uno de las tesis de Wittgenstein sobre la posibilidad de un lenguaje privado, y los tres últimos sobre cuestiones relativas a las descripciones definidas. En uno de ellos se discuten la teoría de Russell y el punto de vista de Strawson.

La obra principal de Villoro en los años sesenta es, con toda seguidad, su libro sobre Descartes, pero la lectura de sus dos colecciones de ensayos se puede hacer como el seguimiento de un itinerario: Páginas filosóficas, el libro de 1962, contiene entre otras materias escritos sobre Dilthey, Marcel y Husserl, pero se cierra con un ensayo titulado "La crítica del positivismo lógico a la metafísica». El libro de 1975 , Estudios sobre Husserl, concluye con una reseña que lleva por título "Fenomenología y filosofía analítica». Hay en la obra ensayística de Villoro, un intento de inte- 
grar elementos en apariencia encontrados, que ya le reprochaba Gaos en 1959, y que parece mantener con vida algún aspecto de su formación inicial. Su libro más importante quiere salir al paso de esa problemática: Crecer, saber. conocer, que no se publicó hasta 1982 , resume el esfuerzo de muchos años de docencia. Es un modelo de investigación analítica que, sin ceñir sus fuentes a una sola tradición filosófica, persigue una red de conceptos fundamentales y precisa sus conexiones sistemáticas; todo en torno a varios tipos de conocimiento y a sus relaciones con la razón práctica.

El tercero de los miembros del primer comité de Crítica -que es el mismo autor de esta nota-, llegó a la filosofía analítica un poco más tarde que los dos anteriores, pero lo hizo por el mismo camino. Dianoia guarda en los años sesenta sus artículos sobre Husserl, Nicolai Hartmann y Heidegger, el último de los cuales da entrada a sus preocupaciones sobre filosofía del lenguaje. En 1967 se imprime el primero de los textos reunidos después en $L a$ filosofía y las actitudes morales, libro que recoge la línea de Brentano y de Husserl para reanudarla con la dirección analítica, y vuelve a plantear la función moral de las concepciones del mundo, con independencia de la tarea filosófica concebida como análisis conceptual. Un punto que da lugar a su discusión con Salazar Bondy, publicada en 1969 por la Universidad de Kansas, y que se mantiene, aunque de manera menos visible, en otros textos de Ensayos filosóficos y de Enseñanza y filosofía. En 1985, Salmerón y Rabossi publicaron un volumen de Etica y análisis, compilación de estudios sobre lenguaje y justificación moral, con textos de Moore, Strawson, Stevenson, Hare, Searle, Foot, Baier y Gilbert Harman, entre otros.
De la generación que sigue inmediatamente a los tres primeros editores de Critica, cabría decir algo parecido en cuanto a formación y trayectoria: Roberto Caso Bercht, Hugo Padilla y Wonfilio Trejo, asistieron a cursos de Gaos y Robert $S$. Hartman, pero también de Villoro y de Rossi. Los primeros escritos de Roberto Caso fueron sobre teoría del valor, pero luego se consagró a la lógica, después de una estancia en Berkeley con el grupo de Tarski, principalmente en la Universidad Autónoma Metropolitana. En esta institución, Roberto Caso promovió una maestría en lógica y filosofía de la ciencia, a la que estuvieron asociados en un cierto momento $\mathrm{C}$. Ulises Moulines, Ignacio Jané, Susana Berestovoy, Mario Otero, Luis Villoro y León Olivé.

Las primeras publicaciones de Hugo Padilla fueron precisamente sobre Husserl, pero después se dedicó también a problemas de lógica; en 1972 dio a la imprenta un volumen con traducciones de Frege: Conceptografia. Los fundamentos de la aritmética y otros estudios filosóficos.

Wonfilio Trejo, fallecido hace poco más de dos años, dejó una obra escrita más amplia y de mayor significación. Había enseñado epistemología en varias universidades mexicanas - la $\mathrm{Ve}$ racruzana, la de Nuevo León y la Nacional Autónoma de México. Después de sus primeras publicaciones, entre ellas un libro sobre Dilthey, y al margen de una antología de ética destinada a la enseñanza, se consagró a la investigación sistemática de problemas del conocimiento. Fenomenalismo y realismo, su libro póstumo, aborda temas fundamentales a partir de los dásicos y somete a análisis concepciones contemporáneas, hasta proponer una posición propia a cuestiones de la percepción y del conocimiento empírico. Un libro anterior; que reúne sus ensayos epistemológicos 
publicados entre 1961 y 1972, descubre la congnuencia de sus preocupaciones, a la vez que la amplitud de su trayectoria. En esos ensayos, se ocupa de Husserl, Sartre, Merleau-Ponty, Russell y Wittgenstein.

16. Ya no sería posible decir nada parecido de las promociones siguientes cuya formación filosófica fue muy distinta y ya no incluyó, como pieza fundamental, el estudio de la fenomenología. Su producción, además comienza después de los años sesenta, que es justo la fecha señalada como límite a esta nota, y por eso ya no puede ser tratada aquí.

En las publicaciones citadas al comienzo de la nota, y en el informe del Instituto de Investigaciones Filosóficas de 1978 , se puede hallar alguna noticia acerca de la mayor parte de los miembros de estas promociones; aunque en el informe aparezcan nombrados apenas como recién ingresados al Instituto, o como becarios del mismo, o cumpliendo estancias postdoctorales en universidades extranjeras. En todo caso, ellos han sido los responsables del desarrollo del análisis filasófico en México en los últimos dos decenios: de la amplitud con la cual los métodos analíticos han llegado, entre nosotros, al tratamiento de cuestiones prácticas, en el orden moral y en el orden político; de una visión más comprensiva de la historia de la ciencia y de los procesos de producción y validación de los conocimientos; e incluso, de lo que ahora se puede empezar a llamar la recepción de la teoría crítica.

Se justifica, sin embargo, hacer un par de excepciones. La primera, con la mención de Hugo Margain, cuyas publicaciones corresponden a los años 1969 a 1977. Margain se había incorporado brillantemente a la vida filosófica mexicana al concluir estudios en $\mathrm{Ox}$ - ford $y$, con sus trabajos de aquellos años, preparó un libro que apareció en 1978, poco después de su muerte, acaecida apenas unos meses más tarde de su designación como director del Instituto de Investigaciones Filosóficas. El libro, Racionalidad, lenguaje y filosofia. discute la cuestión de la racionalidad desde diversos ángulos, que se conectan con problemas' lógicos de la teoría del signilicado, el lenguaje de lo mental, la explicación y la causalidad. $Y$ se orienta todo en la perspectiva de una epistemología naturalista, a la manera de Ouine y Gilbert Harman.

La segunda excepción tiene que ver con los filósofos no mexicanos que durante la década de los años sesenta y los primeros años de la siguiente, visitaron México, en razón de cursos o de encuentros académicos. La mayor parte de ellos - los que se conectaron con el Instituto de Investigaciones Filosóficas-, están registrados en el citado informe. Pero ahora es indispensable hacer mención de quienes permanecieron lapsos mayores -un año o más, por ejemplo. Es el caso de Roberto Vernengo, que estuvo adscrito a la Universidad Autónoma Metropolitana y en ese tiempo publicó, además de sus colaboraciones en revistas, estudios sobre interpretación jurídica y conceptos dogmáticos. También el caso de Otero, Bunge y $\mathrm{C}$. Ulises Moulines, que por un tiempo formaron parte del Instituto de Investigaciones Filosóficas, aunque hicieron labor docente también en otras instituciones. Otero publicó en México, además de articulos, sus dos aproximaciones a la filosofía de la ciencia y su introducción a la dialéctica de Galeno. Mario Bunge, que permaneció en el Instituto entre 1975 y 1976 , fundó y dirigió en ese tiempo la Sociedad Mexicana de Epistemología, y preparó además diversas publicaciones. Su libro Epistemologia, de 1980, recoge parte de 
sus cursos y conferencias mexicanas; pero también trabajó en ese tiempo en uno de los volúmenes de su Tratise on Basic Philosophy.

En los mismos años vino a México el venezolano Ulises Moulines, que permanecio entre nosotros un tiempo más largo y cumplío una labor docente de muy dilatados frutos - de la cual no podemos dar cuenta aquí. Una labor que quedo interrumpida con su cambio a la Universidad de Bielefeld y, después, a la de Berlín. Parte de su producción mexicana está incorporada en su libro de 1982, Exploraciones metacientificas.

17. El caso de Bunge y el de Moulines, como antes el de Garzón Valdés y el de Castañeda, que han cumplido buena parte de su formación y de su obra fuera de la region, han permitido, sin embargo, la alusión indispensable para incorporar sus nombres a la relación que presenta esta nota. Otros casos apenas han podido ser aludidos, como los de Coffa, Gomez y Cordero, y el del español José Ferrater Mora, parte de cuya obra es inseparable de la vida filosófica latinoamericana. En cambio, otros no han podido ser nombrados, como Ignacio Angelelli, desde hace muchos años profesor en Austin, Texas. A pesar de su vinculación, por un tiempo, a la Universidad de Buenos Aires, Angelelli no pertenece a ninguno de los grupos o promociones registrados. Tampoco fueron nombrados Jorge Gracia y Ernesto Sosa, ambos nacidos en Cuba pero cuya formación y actividad profesional ha tenido siempre como base el Canadá y los Estados Unidos.

Pues bien, ahora es indispensable decir que sin ellos el cuadro trazado hasta aquí del análisis filosófico en América Latina es incompleto. Sin excepción, todos han mantenido una relación permanente con los centros latinoamerica- nos de actividad filosófica - no sólo con sus respectivos países de origen. Su presencia en las publicaciones periódi cas, su participación en reuniones académicas y su propia influencia filosófica es un elemento esencial en las tareas normales de nuestros países. Pero es indispensable decir algo más: una manera de hacer filosofía que, en principio se entiende a sí misma como tarea colectiva, deja poco espacio para las peculiaridades locales $\mathrm{y}$ personales $\mathrm{y}$ hace más fácil la comunicación y la vida internacional.

En este sentido, una caraclerística del análisis en América Latina - si se hiciera la comparación con otras comientes filosóficas - ha sido la mayor vinculación de los filósofos latinoamericanos entre sí y su mayor participaciôn en la vida filosófica internacional. Es verdad que en esto han ayudado los tiempos, no tanto por la facilidad de las comunicaciones como por las crisis económicas y políticas.

Pero, tal vez, el análisis filosófico en América Latina no debiera ser tratado como una escuela o corriente filosófica entre otras, aunque en un cierto momento él mismo hubiera trazado fronteras con energía y privilegiado ciertos temas y tradiciones. Entre los años cuarenta y cincuenta - y todavía al comienzo de la década siguiente-, el contexto polémico y los primeros contactos con el empirismo y la lógica moderna favorecieron esa disposición. Hemos visto, sin embargo, que no se trató nunca de rescatar o de volver a una tradición olvidada -aunque en el siglo XTX hubiéramos tenido un filósofo empirista como Andrés Bello. Se trataba más bien de un reclamo en el nivel de las exigencias metodologicas y del estilo; se trataba de una reacción en contra de las posiciones historicistas extremas y de una manera de dramatizar los problemas metafísicos - sin volver, por su- 
puesto, a posiciones dogmáticas. Era un esfuerzo por concretar los planteamientos, afinar el perfil de los conceptos y atender al juego limpio de los argumentos, esto es, al peso que cada argumento tiene por sí mismo y en relación a la cuestión planteada - no al peso que pudiera derivar de su lugar en una cadena de conexiones sistemáticas.

Esta caracterización explica que el análisis encontrara acogida entre los fcnomenólogos, y también entre los lógicos y los investigadores científicos. Explica también la oposición de los heideggerianos $y$, en segundo lugar, de los escolásticos y los marxistas. Y nos permite interpretar correctamente el sentido del cambio de rumbo de la actividad filosófica. Dejar a un lado la simple especulación y tratar de acercarse al ideal husserliano de una filosofía científica, en la mayor parte de los países de América Latina era una forma de continuidad histórico-filosófica. Se dio tam- bién una conciencia de innovación y de ruptura, si no precisamente de una tradición, sí de una forma defectuosa de la curiosidad filosófica. Desde España, Ortega y Gasset había impuesto esa li* mitación, al margen de la cual sólo parecían sobrevivir la escolástica y el marxismo. En México, la presencia del pragmatismo se había dejado sentir al comienzo de los años veinte, pero de una manera superficial, porque enseguida fue restaurada la dieta filosófica a base de Bergson y la literatura escrita en lengua alemana. En cierto modo, había una clara conciencia de esa limitación, como lo prueban muchas expresiones de Gaos y su diálogo polémico con Frondizi - desde los años cuarenta. Lo que se vino a añadir después fue solamente la conciencia de que aquella dieta era una causa grave de enfermedad filosófica: era como nutrir el pensamiento sólo con una clase de ejemplos.
1. En razón de su brevedad, esta nota prescinde de información bibliografica, salvo algunas indicaciones incompletas que se dan en el texto. Pero no puede dejar de sugerir la lectura de un intento reciente por registrar la complejidad de los movimientos filosoficos contemporáneos en América Latina, en The Philosophical Forum, a Quarterly (Vol. XX, Nos. 1-2, Fall-Winter 1988-89). Tampoco puede dejar de indicar su deuda con J. Gracia, E.
Rabossi, E. Villanueva y M. Dascal, editores de $E l$ andisis filosofico en América Latina, Fondo de Cultura Económica, México 1985. La versión inglesa de este libro apareció en Reidel Publishing Co. en 1984. El autor quiere expresar además su agradecimiento a algunos amigos y colcgas: Ernesto Garzón Valdes, Juan Nunio, Alfonso Gómez-Lobo, Rubén Sierra Mejía y Mario Otero. 